(2) Open Access Full Text Article

REVIEW

\title{
Assessment and Monitoring of Patients with Immune-Mediated Thrombotic Thrombocytopenic Purpura (iTTP): Strategies to Improve Outcomes
}

This article was published in the following Dove Press journal: Journal of Blood Medicine

\author{
Selin Kucukyurt \\ Ahmet Emre Eskazan (iD \\ Division of Hematology, Department of \\ Internal Medicine, Cerrahpasa Faculty of \\ Medicine, Istanbul University-Cerrahpasa, \\ Istanbul, Turkey
}

Background: Acquired or immune-mediated TTP (iTTP) is a life-threatening thrombotic microangiopathy, characterized by the presence of microangiopathic hemolytic anemia and severe thrombocytopenia, and a variable degree of ischemic organ damage, related to a severe deficiency of ADAMTS13, which is a serine metalloprotease necessary for cleavage of large vWF multimers. There has been a dramatic decrease in mortality rates with the recognition of the pathophysiology of iTTP over the years. Although therapeutic plasma exchange (TPE) together with corticosteroids are the backbone of the upfront treatment of patients with iTTP with successful outcomes, patients may remain refractory and/or relapse following an initial response to this treatment.

Methods: We performed a review regarding the pathogenesis, diagnosis, treatment strategies, monitoring, and prognosis of iTTP.

Results: There are several new treatment strategies, which can be used among these patients, helping in improving outcomes of iTTP. Rituximab has been shown to be a safe and effective adjunct to TPE, especially in patients with refractory and/or relapse as well as it is increasingly used preemptively to prevent exacerbation or recurrence. Recently, caplacizumab, a nanobody targeting vWF, was approved as an addition to the current regimen of TPE and immunomodulation for patients of iTTP.

Conclusion: Specific predictors of relapse in patients in remission can be relevant for an optimal patient management. Different models including ADAMTS13 biomarkers can provide a new screening strategy to identify patients who may predict outcomes and the risk of relapse, benefit from preemptive therapy prior to relapse.

Keywords: immune-mediated thrombotic thrombocytopenic purpura, ADAMTS13, caplacizumab, monitorization, outcome, TTP

\section{Introduction}

Thrombotic microangiopathies (TMAs) are a group of disorders, which are generally associated with thrombocytopenia and microangiopathic hemolytic anemia (MAHA). ${ }^{1}$ Thrombotic thrombocytopenic purpura (TTP) is a TMA which can be divided into 2 as hereditary (Schulman-Upshaw syndrome) and acquired or immune-mediated TTP (iTTP), and iTTP can be further divided into two as primary and secondary.

Secondary TTP can be associated with various disorders including connective tissue disease (such as systemic lupus erythematosus, Sjogren's syndrome, and rheumatoid arthritis), infectious agents (such as HIV infection, cytomegalovirus
Correspondence: Ahmet Emre Eskazan Istanbul University-Cerrahpasa,

Cerrahpasa Faculty of Medicine,

Department of Internal Medicine,

Division of Hematology, Fatih, Istanbul, Turkey

Tel +905337227376

Fax +902125897934

Email emre.eskazan@istanbul.edu.tr
Journal of Blood Medicine 2020:1 I 319-326 
infection), drugs (including ticlodipine, quinine, gemcitabine and mitomycin) and pregnancy. ${ }^{2}$ Primary iTTP occurs due to acquired deficiency of ADAMTS13, a serine metalloprotease required for the cleavage of ultra-large von Willebrand factor (vWF) multimers. ${ }^{1,3}$ iTTP can be distinguished from other causes of MAHA by severe ADAMTS13 deficiency and the presence of an inhibitor (autoantibody directed against ADAMTS13). It is an acute rare syndrome and medical emergency that can rapidly show a fatal course if the diagnosis and/or treatment is delayed. ${ }^{4,5}$

Although TPE together with corticosteroids are the cornerstone of the upfront treatment of iTTP generally with successful outcomes, patients may remain refractory and/or relapse following an initial response to this treatment. Twice-daily plasma exchange and some agents including rituximab and caplacizumab can be utilized in the management of refractory iTTP. ${ }^{4}$ On the other hand, exacerbation/relapse may occur during the follow-up in about half of patients. All patients with a relapsed iTTP should be treated promptly with TPE and corticosteroids and rituximab and/or caplacizumab can be used in selected patients. Also, splenectomy may be an option, especially for those with multiple relapses. ${ }^{4}$

Specific predictors of relapse in patients in remission can be relevant for an optimal patient management. ADAMTS13 testing may also provide prognostic information, with lower levels of ADAMTS13 and higher levels of anti-ADAMTS13 antibodies associated with higher relapse rates. Therefore, close follow-up of patients, use of ADAMTS13-based objective tests (ADAMTS13 activity, inhibitor, autoantibody, conformational change) in follow-up and interpretation of these tests are important to identify patients who may predict outcomes and the risk of relapse, benefit from preemptive therapy with rituximab prior to relapse. ${ }^{6,7}$

This review mainly focuses on the assessment and monitoring of patients with primary iTTP together with overviewing the strategies to improve outcomes.

\section{Pathogenesis of iTTP}

In iTTP, an autoantibody against ADAMTS13 is produced, which leads the neutralization of ADAMTS13 that physiologically cleaves the ultra-large multimers of vWF, and the severe deficiency of this protease is the major cause of iTTP. When left uncleaved, ultra-large vWF multimers bind firmly to platelets and form aggregates capable of occluding arterioles especially where circulation is slower.
Fatal organ damage due to microvascular thrombi, bleeding findings due to thrombocytopenia and findings related to mechanical hemolysis in erythrocytes result in the clinical presentation of iTTP. ${ }^{3,8}$

Microthrombi in iTTP are associated with the accumulation of inactivated platelets that do not provoke coagulation and fibrin accumulation, and they can be found in all tissues; lesions are usually seen in the heart, pancreas, kidney and brain, but are rare in the lungs and liver. ${ }^{9,10}$

\section{Diagnosis}

\section{Clinical Diagnosis}

TTP was first identified in a 16-year-old young girl with fatal thrombotic microangiopathy by Moschcowitz in 1924. ${ }^{9}$ The typical clinical presentation and pentad of TTP were first defined by Amorosi and Ultman in 1964, which includes fever, MAHA, thrombocytopenia, renal dysfunction and changes in mental status. ${ }^{11}$ As the onset of TTP may be insidious with nonspecific symptoms such as myalgia, arthralgia, fatigue, malaise, it may be also present with symptoms related to microvascular organ thrombosis such as focal neurological abnormalities and chest pain.

The presence of MAHA and thrombocytopenia is essential for the diagnosis of iTTP. iTTP can be diagnosed with clinical history, physical examination and routine laboratory tests, and notably peripheral blood smear. Peripheral blood smear features consistent with MAHA include fragmented red blood cells - schistocytes (which are erythrocytes broken down by microthrombi is typical in peripheral blood smear) with a threshold value of $1 \%$, polychromasia, and anemia. ${ }^{2,3}$ At the time of admission, platelet count is typically below $30 \times 10^{9} / \mathrm{L}$ and hemoglobin level is usually $8-10 \mathrm{~g} / \mathrm{dL}$. Bleeding due to thrombocytopenia may occur.

Laboratory findings related to non-immune hemolysis (negative direct Coombs test, but increased indirect bilirubin, increased lactate dehydrogenase (LDH), decreased haptoglobin and reticulocytosis) and normal routine coagulation parameters strongly support the diagnosis. LDH levels may increase due to both hemolysis and tissue ischemia. Coagulation tests are generally normal. The remaining features of the classical pentad including acute renal damage, neurological abnormalities and fever may less often occur in late presentation. ${ }^{2}$

Bendapudi et $\mathrm{a}^{12}$ developed a diagnostic scoring system for the early treatment of patients who were suspected 
of having TTP and could benefit from TPE, since there is no reliable and rapid biological test for the diagnosis of TTP. This scoring system so-called the PLASMIC score is not used to confirm or exclude the diagnosis of TTP, but it is used in order to predict the probability of ADAMTS13 activity of $\leq 10 \%$ in adults. It is based on clinical and biological data obtained at the admission of patients and consists of seven parameters; platelet count $<30 \times 10^{9} / \mathrm{L}$ at the time of diagnosis, combined hemolysis variables (reticulocyte count $>2.5 \%$, indirect bilirubin $>2.0 \mathrm{mg} / \mathrm{dL}$, undetectable haptoglobin), absence of active neoplasia, absence of organ or stem cell transplantation history, mean corpuscular volume $<90 \mathrm{fL}$, INR $<1.5$, and serum creatinine levels $<2.0 \mathrm{mg} / \mathrm{dL}$. Each parameter is scored as 1 point, and the total score ranges from 1 to 7 . The PLASMIC score stratifies patients into low-risk (0-4), intermediate-risk (5), and high-risk (6-7) categories. If the PLASMIC score is high (6-7), ADAMTS13 activity is $\leq 10 \%$ with a sensitivity of $91 \%$.

\section{Laboratory Tests}

The diagnosis of iTTP depends on ADAMTS13 testing especially in atypical presentations. iTTP has severe ADAMTS13 deficiency in the acute episode, with or without symptoms of tissue damage. However, life-threatening microvascular thrombosis such as cardiac injury and stroke are sometimes seen in patients with ADAMTS13 activity below $10 \%$ of normal before developing MAHA or thrombocytopenia. ${ }^{13-15}$

Having said that, although ADAMTS13 assays are crucial and help to confirm the diagnosis of iTTP, this is not always straightforward especially in the developing countries, and physicians should not wait for the determination of ADAMTS13 activity to make the diagnosis of iTTP. ${ }^{16}$ It may take several days to get the test results, and this could delay the initiation of TPE, which may result in morbidity and/or mortality. Thus, diagnostic work-up should be planned as TPE is started in a patient with possible iTTP. ${ }^{17}$ The tests used for the diagnosis of iTTP are shown in Table 1.

Although some are not routinely performed, ADAMTS13 antigen, activity, neutralizing and nonneutralizing antibodies and gene mutations can be investigated. The results must be obtained before plasma therapy or any kind of blood product is administered in order to interpret them correctly. ADAMTS13 activity is usually diagnostic. It has been reported that ADAMTS13 activity may decrease in non-TTP conditions such as uremia, inflammatory
Table I The Diagnostic Tests for Suspected TTP (Adapted from References 13 and 17)

-Complete blood count
-Peripheral smear, reticulocyte count
-Serum biochemistry and creatinine
-Serum LDH, bilirubin, and haptoglobin levels
-Blood group and antibody screen, direct antiglobulin (Coombs) test
(DAT)
-Coagulation tests (aPTT, PT, fibrinogen, D-dimer)
-ADAMTS antigen, activity, inhibitor
-HIV, hepatitis B virus, and hepatitis C virus serology
-Autoantibody screen: ANA, anti-ds DNA, anticentromere
antibodies, anti-cardiolipin antibody, antiphospholipid ab, anti-
Phospholipid ab
-Pregnancy test
-Folic acid, vitamin BI2
-Serum troponin level and ECG
-Cranial imaging if neurological symptom is present
-ADAMTSI3 gene mutations if there is suspicion of hereditary TTP
-Blood culture and other foci of suspected infection should be
examined in patients with fever or symptom suggestive of systemic
infection
-Stool studies (cultures, toxin testing) for Shiga toxin or Shiga toxin-
producing organisms
-Required imaging if suspicious symptoms and signs of malignancy
-Question drug history

conditions, post-operative and pregnancy, but ADAMTS13 activity is usually $>10 \%$ in these disorders. ${ }^{18}$ Collagenbinding assays and the FRETS-VWF73 assay are the most commonly used reference methods. FRETS-WWF73-based assay is frequently utilized of one of the reference methods for ADAMTS13 activity measurement. ADAMTS13 antigen levels can be measured by an ELISA. Neutralizing antiADAMTS13 antibodies are detected and quantified by the ADAMTS13 functional inhibitor assay, and they are based on ADAMTS13 activity measurement by mixing patient plasma and control plasma in a 1:1 ratio. Rather than interfering with ADAMTS13 proteolytic activity, nonneutralizing antibodies promote ADAMTS13 clearance from plasma. Non-neutralizing antibodies can be also demonstrated by ELISA or Western blotting. ${ }^{8,15,19}$

There is an ADAMTS13 inhibitor circulating in plasma in $94-97 \%$ of iTTP cases. The majority of these inhibitors are antibodies that severely inhibit ADAMTS13 activity. Approximately $10 \%$ of circulating antibodies also bind to ADAMTS13, causing the molecule to rapidly clear from circulation (increased clearance). The result is severe ADAMTS13 deficiency. Most of these antibodies are of IgG type. However, IgM and $\operatorname{IgA}$ antibodies can also be 
detected. However, the inhibitor cannot always be detected. This may be due to very low inhibitor titer for in vitro measurement, inhibitor being a non-neutralizing, patient with hereditary TTP, neutralization of the inhibitor with anti-idiotype antibodies found in ADAMTS13 or blood product transfusions. ${ }^{19-21}$

ADAMTS13 generally circulates in a folded conformation, but it circulates in an open conformation during acute episodes of iTTP. However, open ADAMTS13 is not only a hallmark of acute iTTP, and anti-ADAMTS13 autoantibodies from iTTP patients induce an open ADAMTS13 conformation. An open ADAMTS13 conformation differentiates TTP from other TMAs, is a biomarker for subclinical iTTP and could become a key component in TTP management. $^{7}$

\section{Treatment Options of iTTP}

If untreated, TTP has a mortality rate of as high as $90 \%$. TPE was found to be an effective treatment, and it became the standard of care in 1991..$^{5}$ If there is significant clinical suspicion (the presence of MAHA + thrombocytopenia not associated with another cause), then treatment should be started after a presumptive diagnosis of iTTP. ${ }^{13}$ In iTTP, it is aimed to replace the lacking ADAMTS13 with TPE and to remove circulating autoantibodies in the patient serum. ${ }^{22}$ The recommended replacement dose for TPE is usually $1.5 \times$ plasma volume exchange for the first procedures, followed by $1.0 \times$ patient plasma volume thereafter ( 40 to $60 \mathrm{~mL} / \mathrm{kg}$ ). All plasma products (quarantine fresh frozen plasma, plasma virally attenuated with solvent/detergent, or a psoralen - derivative (amotosalen)) appear to be equally effective. ${ }^{13,23}$ Complications related to TPE are mostly related to central venous catheter such as infection, sepsis, hemorrhage, catheter obstruction, venous thrombosis. Infrequently, plasmarelated complications such as anaphylaxis, serum sickness, and transfusion-related acute lung injury (TRALI) are observed. High volume plasma exchange can cause hypotension, arrhythmia, hypokalemia, metabolic alkalosis and hypocalcemia. ${ }^{23,24}$

Corticosteroids with TPE are routinely added to the initial treatment of patients with suspected iTTP. $^{23}$ Corticosteroids reduce ADAMTS13 inhibitor production, thereby reducing the number of TPE, required and accelerating recovery as a result. Decreased cytokine production or decreased autoantibody-mediated clearance of ADAMTS13 may also contribute to this effect. There is no study with randomized trial of the use of corticosteroids in the treatment of iTTP. The use of glucocorticoids and rituximab in combination with TPE treatment decreases the frequency of TPE to achieve remission. ${ }^{25}$ The dose and route of corticosteroids administered varies depending on the presentation of the disease. ${ }^{26}$

TPE is continued until the diagnosis of TTP is excluded, or until an explanatory alternative diagnosis is made, and until response to treatment is achieved. If there is no increase in platelet count in the first few days of the treatment; then refractory disease, alternative primary diagnosis, or development of complications (e.g. catheterrelated sepsis) must be examined as a possibility. ${ }^{6,25,27,28}$

Clinical response to treatment is defined as the normal platelet count (above $150 \times 10^{9} / \mathrm{L}$ ) for 2 consecutive days, normalizing LDH and clinical recovery. Clinical remission is that the platelet count is within normal limits for the first 30 days after TPE is stopped. Exacerbation is the recurrence of thrombocytopenia and an increased LDH level within the first 30 days after TPE is stopped (typically platelet count $<150 \times 10^{9} / \mathrm{L}$ ). Relapse is the recurrence of TTP acute episode following remission. Refractory TTP is described as persistent thrombocytopenia, lack of a sustained platelet count increment or platelet counts of $<50 \times 10^{9} / \mathrm{L}$ and a persistently raised LDH level despite five plasma exchanges and steroid treatment. ${ }^{2,29}$ Some patients may have either insufficient or transient response or no response to initial therapy, including TPE and corticosteroids. In case of relapse/refractory disease, TPE is restarted, if stopped. TPE frequency or density can be increased and/or corticosteroid therapy may be intensified. If the patient has not previously received rituximab therapy, rituximab can be added to treatment. Caplacizumab may be administered, if it has not been previously taken or discontinued. ${ }^{6,30}$

Rituximab is a chimeric monoclonal antibody directed against the B-cell lineage-specific CD20 antigen. Rituximab therapy increases the level of plasma ADAMTS13 by alleviates the production of anti-ADAMTS13 antibodies. In patients with an episode of refractory TTP, addition of rituximab to TPE and corticosteroids increases response rate. The French TMA reference center reported a higher rate of recovery and a lower relapse rate with rituximab therapy in relapse/refractory disease. Also, in a series of 25 patients with refractory/relapsed TTP, remission was achieved in all patients and none of them relapsed over a median follow-up of 19 months. ${ }^{31-34}$ Because TPE removes plasma and plasma proteins, it will also remove monoclonal antibodies. Thus, the timing of TPE and monoclonal antibodies may be important when used together. Rituximab should be timed to be given after TPE if possible, since rituximab will be purged 
from the circulation with TPE. Rituximab is administered short after TPE promptly to prolong its efficient period until it is removed by the subsequent TPE, and avoiding TPE for at least 12 hours following the administration of rituximab is recommended. $^{23}$ There are potential risks such as rituximabrelated infusion reactions, mucocutaneous reactions, longterm immunosuppression, hepatitis $\mathrm{B}$ reactivation, and progressive multifocal leukoencephalopathy. As per serological results, antiviral prophylaxis for hepatitis B should be initiated before rituximab treatment. ${ }^{35,36}$

Caplacizumab is a humanized bivalent variabledomain immunoglobulin that recognizes human VWF A1 domain and blocks to link the platelet GPIb, reducing platelet aggregation and microvascular thrombosis, also facilitates normalization of the platelet count. In multicenter randomized placebo-controlled clinical trials TITAN (phase II) and HERCULES (phase III), caplacizumab significantly decreased the 30-day recurrence rate, and reduced the rate of the composite endpoint of death, recurrence, and major thromboembolic events, also it is well tolerated with minor bleedings as primary adverse effect. The US FDA and European Medicines Agency approved caplacizumab for adult patients with iTTP as a frontline treatment, in combination with TPE and immunosuppressive therapy. ${ }^{4,37-39}$ Based on the mechanism of action, caplacizumab is expected to rapidly stop microthrombi formation causing disease complications, but it is not expected to block ADAMTS13 activity and to reduce autoantibody production against ADAMTS13. Patients with persistent ADAMTS13 deficiency quickly relapse when caplacizumab is discontinued. ADAMTS13 monitoring may be useful to decide whether to discontinue treatment after the ADAMTS13 level is elevated. Daily caplacizumab is continued until 30 days after the last TPE session. $^{38,39}$

In patients with relapsed/refractory iTTP, cyclophosphamide, cyclosporine, mycophenolate, vincristine, bortezomib, $\mathrm{N}$-acetylcysteine, and splenectomy can be beneficial. ${ }^{40-46}$

In addition to the aforementioned treatment approaches, caution should be exercised for other potential complications of TTP treatment. Although platelet transfusion is usually contraindicated in TTP as it can lead to life-threatening thrombotic events, platelet transfusion may be required in patients with severe thrombocytopenia, who have clinically life-threatening bleeding or require urgent surgical intervention. $^{23,47}$ Individuals with neurological findings or high troponin levels should be evaluated by the relevant departments. Thrombocytopenia is not protective against thrombosis. Venous thromboembolism prophylaxis may be required in some patients. When platelet count is greater than $50 \times 10^{9} / \mathrm{L}$, low-dose aspirin is recommended to be initiated in patients with TTP, and in addition to that folic acid supplementation should be given to all patients due to hemolytic status. ${ }^{23}$ When TPE cannot be initiated immediately, simple plasma infusion can be performed in iTTP for preventing a delay in the treatment. ${ }^{48}$

\section{Monitorization of Patients with iTTP}

The most important problem in iTTP is the risk of unpredictable, life-threatening relapse which may result of TPE complications, ICU hospitalization or death. Up to $\% 40$ of patients with iTTP who achieve clinical response to firstline therapy experience relapse. ${ }^{13}$ TTP relapses are usually recognized faster than the first episode, because the patient and clinician are familiar with TTP symptoms. Any disease, surgery, major trauma can cause TTP relapse. The risk of TTP relapse increases during pregnancy. Similarly, to the first episode of TTP, platelet count should be measured immediately and rapid interventions for thrombocytopenia should be performed if symptoms (bruising, dark urine, neurologic symptoms or unexplained severe fatigue) develop. ${ }^{3,49}$ Therefore, particularly patients with a persistently low ADAMTS13 activity should avoid external triggers (infections, pregnancy, surgery) to avoid relapsing TTP. $^{49}$

Currently, clinical factors and laboratory biomarkers that could reliably predict the iTTP exacerbation and/or relapse are insufficient. ADAMTS-13 activity monitoring during the follow-up after the acute episode allows discovering patients in remission with persistent undetectable protease activity. ${ }^{50}$ Several studies have demonstrated that patients with low ADAMTS-13 activity plus high antiADAMTS13 antibody titer at remission had an increased risk of relapse. ${ }^{51-54}$ Also, Schieppati and colleagues ${ }^{54}$ found that patients requiring a shorter time to achieve clinical remission with TPE first-line treatment (i.e. $<13$ days) showed a significantly lower risk of relapse. In addition, Sui et $\mathrm{al}^{55}$ recommended that plasma ADAMTS13 activity, ADAMTS13 antigen, and antiADAMTS13 IgG levels be tested 3 to 7 days after the initiation of TPE and at clinical response/remission. They demonstrated the critical role of longitudinal assessments of plasma ADAMTS13 biomarkers including ADAMTS13 activity, ADAMTS13 antigen, and anti-ADAMTS13 IgG in predicting iTTP exacerbation and/or recurrence. 
In clinical remission, although a persistent severe ADAMTS13 deficiency may predict the risk of relapse, a severe ADAMTS13 deficiency does not necessarily induce a TTP episode and additional triggers are required and need to be identified, knowing the fact that some patients who have low ADAMTS13 activity do not clinically relapse. ${ }^{51-54}$ Conditions that might increase plasma VWF levels including inflammation, sepsis, or pregnancy may accelerate acute TTP episodes. Besides, a reduced DNase activity in TTP patient plasma was demonstrated to result in the persistence of neutrophil extracellular traps and could act as the second hit needed to provoke an acute TTP episode, since human neutrophil peptides are also able to inhibit ADAMTS13 activity in vitro. ${ }^{49,56}$

Rituximab is administered as a preemptive therapy for the persistence of severe ADAMTS13 deficiency associated with an increased risk of relapsed during hematological remission to ensure normalization of ADAMTS13 activity and to prevent overt relapse. ${ }^{33,34}$ Current studies also focus on the ideal dose and the treatment scheme of rituximab in iTTP. ${ }^{57}$

The risk of exacerbation is highest in the first week after TPE is stopped. After stopping TPE, patients are monitored by platelet count, blood film, LDH and weekly plasma ADAMTS13 antigen, activity, anti-ADAMTS13 IgG measurements, especially 2-3 times a week for the first week, and then followed by once a week for the first month. ${ }^{2,8,21}$ ADAMTS13 is an auxiliary indicator of potential relapse, but the timing of relapse is variable. The risk of relapse is high in the first two years, and asymptomatic patients are followed up with ADAMTS13 antigen, activity and autoantibodies every 3 months for the first 2 years. After the first 2 years, ADAMTS13 is followed-up annually. However, relapse may occur at any time, therefore, iTTP patients should be followed for lifelong. ${ }^{2,3,13}$

\section{Prognosis and Long-Term Complications}

Relapse is one of the most important and feared issues following recovery from iTTP. Furthermore, in patients with TTP, the risk of developing major depression, minor cognitive impairment, autoimmune disorders especially SLE, new-onset hypertension and abnormal renal function is greater than in general population. ${ }^{58}$ Depression may be the most common disorder following recovery from TTP and it can be hazardous because this condition often overlooked. The clinical features, treatment, and response to treatment is similar with the non-TTP group. In contrast to depression, there are no specific treatment options for cognitive impairment. Among women who had a pregnancy following recovery from TTP, relapse during pregnancy or postpartum period is uncommon. The most important result for the impact of morbidities following recovery from TTP is decreased survival. Deaths may be related to morbidities caused by the previous TTP episode(s). Older age is associated with increased mortality in iTTP and atypical clinical features as neurological presentation may cause delay in diagnosis. Senility negatively impacts the life expectancy. Following recovery from TTP, lifelong careful followup and a comprehensive review of psychiatry, obstetrics, and internal medicine are crucial. ${ }^{58-60}$

\section{Conclusion}

iTTP is a medical emergency and a chronically relapsing, rare and life-threatening autoimmune disorder. Markedly low levels of ADAMTS13, which is pathognomonic for iTTP, results in microthrombi formation which leads to end-organ ischemia and damage, is treated with TPE and immune suppression. Despite the developed scoring system, the diagnosis of iTTP can still be challenging, where rapid recognition is crucial. Currently, the standard of care is still TPE and corticosteroids which reduce the duration of ADAMTS13 deficiency in iTTP. Rituximab which is also frequently used in the treatment of iTTP, may not reduce the number TPE procedures required to induce remission, improves relapse-free survival. Caplacizumab improves iTTP prognosis significantly at the very early, critical step of the disease. However, real-life data are needed for this agent about its efficacy and side effects. The high cost of caplacizumab is one of the disadvantages of the drug.

The monitoring of iTTP for unpredictable relapse is complicated as there is no specific biomarker other than ADAMTS13. For the treatment of patients with iTTP, safer and more non-invasive alternatives to TPE are required. Targeted therapy against $\mathrm{vWF}$ and antiADAMTS13 antibodies is emerging strategies for treatment of iTTP. The results of clinical trials using recombinant ADAMTS13 and N-Acetylcysteine are still awaited. These innovations and developments will further improve survival and decrease the morbidity and mortality related to iTTP. 


\section{Disclosure}

All authors have no conflicts of interest to declare related to this work.

\section{References}

1. Hosler GA, Cusumano AM, Hutchins GM. Thrombotic thrombocytopenic purpura and hemolytic uremic syndrome are distinct pathologic entities. A review of 56 autopsy cases. Arch Pathol Lab Med. 2003;127(7):834-839.

2. Scully M, Cataland S, Coppo P, et al. Consensus on the standardization of terminology in thrombotic thrombocytopenic purpura and related thrombotic microangiopathies. $J$ Thromb Haemost. 2017;15 (2):312-322. doi:10.1111/jth.13571

3. Sadler JE. Pathophysiology of thrombotic thrombocytopenic purpura. Blood. 2017;130(10):1181-1188. doi:10.1182/blood-2017-04-636431

4. Elverdi T, Eskazan AE. Caplacizumab as an emerging treatment option for acquired thrombotic thrombocytopenic purpura. Drug Des Devel Ther. 2019;13:1251-1258. doi:10.2147/DDDT.S134470

5. Rock GA, Shumak KH, Buskard NA, et al. Comparison of plasma exchange with plasma infusion in the treatment of thrombotic thrombocytopenic purpura. Canadian apheresis study group. $N$ Engl $\mathrm{J} \mathrm{Med}$. 1991;325(6):393-397. doi:10.1056/NEJM199108083250604

6. Sayani FA, Abrams CS. How I treat refractory thrombotic thrombocytopenic purpura published correction appears in blood. Blood. 2015;125(25):3860-3867. doi:10.1182/blood-2014-11-551580

7. Roose E, Schelpe AS, Tellier E, et al. Open ADAMTS13, induced by antibodies, is a biomarker for subclinical immune-mediated thrombotic thrombocytopenic purpura. Blood. 2020;136(3):353-361.

8. Masias C, Cataland SR. The role of ADAMTS13 testing in the diagnosis and management of thrombotic microangiopathies and thrombosis. Blood. 2018;132(9):903-910. doi:10.1182/blood-201802-791533

9. Moschcowitz E. Hyaline thrombosis of the terminal arterioles and capillaries: a hitherto undescribed disease. Proc N Pathol Soc. 1924;24:21-24.

10. Nokes T, George JN, Vesely SK, Awab A. Pulmonary involvement in patients with thrombotic thrombocytopenic purpura. Eur J Haematol. 2014;92(2):156-163. doi:10.1111/ejh.12222

11. Amorosi EL, Ultmann JE. Thrombotic thrombocytopenic purpura: report of 16 cases and review of the literature. Medicine. 1966;45 (2):139-160. doi:10.1097/00005792-196603000-00003

12. Bendapudi PK, Hurwitz S, Fry A, et al. Derivation and external validation of the PLASMIC score for rapid assessment of adults with thrombotic microangiopathies: a cohort study. Lancet Haematol. 2017;4(4): e157-e164. doi:10.1016/S2352-3026(17)30026-1

13. Joly BS, Coppo P, Veyradier A. Thrombotic thrombocytopenic purpura. Blood. 2017;129(21):2836-2846. doi:10.1182/blood-201610-709857

14. Sadler JE. What's new in the diagnosis and pathophysiology of thrombotic thrombocytopenic purpura. Hematology Am Soc Hematol Educ Program. 2015;2015(1):631-636.

15. Chiasakul T, Cuker A. Clinical and laboratory diagnosis of TTP: an integrated approach. Hematology Am Soc Hematol Educ Program. 2018;2018(1):530-538. doi:10.1182/asheducation-2018.1.530

16. Eskazan AE, Salihoglu A. Treatment and outcome of primary and secondary thrombotic microangiopathies. Am J Nephrol. 2015;41 (6):427-428. doi:10.1159/000437002

17. Scully M, Goodship T. How I treat thrombotic thrombocytopenic purpura and atypical haemolytic uraemic syndrome. $\mathrm{Br} J$ Haematol. 2014;164(6):759-766. doi:10.1111/bjh.12718

18. Mannucci PM, Canciani MT, Forza I, Lussana F, Lattuada A, Rossi E. Changes in health and disease of the metalloprotease that cleaves von Willebrand factor. Blood. 2001;98(9):2730-2735. doi:10. 1182/blood.V98.9.2730
19. Shelat SG, Smith P, Ai J, Zheng XL. Inhibitory autoantibodies against ADAMTS-13 in patients with thrombotic thrombocytopenic purpura bind ADAMTS-13 protease and may accelerate its clearance in vivo. J Thromb Haemost. 2006;4(8):1707-1717. doi:10.1111/ j.1538-7836.2006.02025.x

20. Rieger M, Mannucci PM, Kremer Hovinga JA, et al. ADAMTS13 autoantibodies in patients with thrombotic microangiopathies and other immunomediated diseases. Blood. 2005;106(4):1262-1267. doi:10.1182/blood-2004-11-4490

21. Kremer Hovinga JA, Vesely SK, Terrell DR, Lämmle B, George JN. Survival and relapse in patients with thrombotic thrombocytopenic purpura. Blood. 2010;115(8):1500-1662. doi:10.1182/blood-2009-09243790

22. Zheng XL, Kaufman RM, Goodnough LT, Sadler JE. Effect of plasma exchange on plasma ADAMTS13 metalloprotease activity, inhibitor level, and clinical outcome in patients with idiopathic and nonidiopathic thrombotic thrombocytopenic purpura. Blood. 2004;103(11):4043-4049. doi:10.1182/blood-2003-11-4035

23. Blombery P, Scully M. Management of thrombotic thrombocytopenic purpura: current perspectives. J Blood Med. 2014;5:15-23.

24. McClain RS, Terrell DR, Vesely SK, George JN. Plasma exchange complications in patients treated for thrombotic thrombocytopenia purpura-hemolytic uremic syndrome: 2011 to 2014. Transfusion. 2014;54(12):3257-3259. doi:10.1111/trf.12917

25. Som S, Deford CC, Kaiser ML, et al. Decreasing frequency of plasma exchange complications in patients treated for thrombotic thrombocytopenic purpura-hemolytic uremic syndrome, 1996 to 2011. Transfusion. 2012;52(12):2525. doi:10.1111/j.1537-2995.2012. 03646.x

26. George JN. How I treat patients with thrombotic thrombocytopenic purpura: 2010 published correction appears in blood. Blood. 2010;116(20):4060-4069. doi:10.1182/blood-2010-07-271445

27. Booth KK, Terrell DR, Vesely SK, George JN. Systemic infections mimicking thrombotic thrombocytopenic purpura. Am J Hematol. 2011;86(9):743-751. doi:10.1002/ajh.22091

28. George JN. Systemic malignancies as a cause of unexpected microangiopathic hemolytic anemia and thrombocytopenia. Oncology (Williston Park). 2011;25(10):908-914.

29. Padmanabhan A, Connelly-Smith L, Aqui N, et al. Guidelines on the use of therapeutic apheresis in clinical practice - evidence-based approach from the writing committee of the American society for apheresis: the eighth special issue. $J$ Clin Apher. 2019;34 (3):171-354.

30. Nguyen L, Li X, Duvall D, Terrell DR, Vesely SK, George JN. Twice-daily plasma exchange for patients with refractory thrombotic thrombocytopenic purpura: the experience of the Oklahoma registry, 1989 through 2006. Transfusion. 2008;48(2):349-357.

31. Scully M, Cohen H, Cavenagh J, et al. Remission in acute refractory and relapsing thrombotic thrombocytopenic purpura following rituximab is associated with a reduction in IgG antibodies to ADAMTS-13. Br J Haematol. 2007;136(3):451-461. doi:10.1111/ j.1365-2141.2006.06448.x

32. Froissart A, Buffet M, Veyradier A, et al. Efficacy and safety of first-line rituximab in severe, acquired thrombotic thrombocytopenic purpura with a suboptimal response to plasma exchange. Experience of the French thrombotic microangiopathies reference center. Crit Care Med. 2012;40(1):104-111. doi:10.1097/CCM.0b013e31822e9d66

33. Lim W, Vesely SK, George JN. The role of rituximab in the management of patients with acquired thrombotic thrombocytopenic purpura. Blood. 2015;125(10):1526-1531. doi:10.1182/blood-2014-10-559211

34. Jestin M, Benhamou Y, Schelpe AS, et al. Preemptive rituximab prevents long-term relapses in immune-mediated thrombotic thrombocytopenic purpura. Blood. 2018;132(20):2143-2153. doi:10.1182/ blood-2018-04-840090

35. Available from: https://www.accessdata.fda.gov/drugsatfda_docs/ label/2009/103705s5299lbl.pdf. Accessed February 20, 2020. 
36. McDonald V, Manns K, Mackie IJ, Machin SJ, Scully MA. Rituximab pharmacokinetics during the management of acute idiopathic thrombotic thrombocytopenic purpura. J Thromb Haemost. 2010;8(6):1201-1208. doi:10.1111/j.1538-7836.2010.03818.x

37. Duggan S. Caplacizumab: first global approval published correction appears in drugs. Drugs. 2018;78(15):1639-1642. doi:10.1007/ s40265-018-0989-0

38. Peyvandi F, Scully M, Kremer Hovinga JA, et al. Caplacizumab for acquired thrombotic thrombocytopenic purpura. $N \mathrm{Engl} \mathrm{J} \mathrm{Med.}$ 2016;374(6):511-522. doi:10.1056/NEJMoa1505533

39. Scully M, Cataland SR, Peyvandi F, et al. Caplacizumab treatment for acquired thrombotic thrombocytopenic purpura. $N$ Engl J Med. 2019;380(4):335-346. doi:10.1056/NEJMoa1806311

40. Yilmaz M, Eskazan AE, Unsal A, et al. Cyclosporin A therapy on idiopathic thrombotic thrombocytopenic purpura in the relapse setting: two case reports and a review of the literature. Transfusion. 2013;53(7):1586-1593. doi:10.1111/j.1537-2995.2012.03944.x

41. Zheng X, Pallera AM, Goodnough LT, Sadler JE, Blinder MA. Remission of chronic thrombotic thrombocytopenic purpura after treatment with cyclophosphamide and rituximab. Ann Intern Med. 2003;138 (2):105-108. doi:10.7326/0003-4819-138-2-200301210-00011

42. Ahmad HN, Thomas-Dewing RR, Hunt BJ. Mycophenolate mofetil in a case of relapsed, refractory thrombotic thrombocytopenic purpura. Eur J Haematol. 2007;78(5):449-452. doi:10.1111/j.16000609.2007.00832.x

43. Öngören S, Salihoğlu A, Apaydın T, et al. Vincristine as an adjunct to therapeutic plasma exchange for thrombotic thrombocytopenic purpura: a single-institution experience. Balk Med J. 2018;35(6): 417-421.

44. Eskazan AE. Bortezomib therapy in patients with relapsed/refractory acquired thrombotic thrombocytopenic purpura. Ann Hematol. 2016;95(11):1751-1756. doi:10.1007/s00277-016-2804-x

45. Rottenstreich A, Hochberg-Klein S, Rund D, Kalish Y. The role of $\mathrm{N}$-acetylcysteine in the treatment of thrombotic thrombocytopenic purpura. J Thromb Thrombolysis. 2016;41(4):678-683. doi:10.1007/ s11239-015-1259-6

46. Kappers-Klunne MC, Wijermans P, Fijnheer R, et al. Splenectomy for the treatment of thrombotic thrombocytopenic purpura. $\mathrm{Br}$ $J$ Haematol. 2005;130(5):768-776. doi:10.1111/j.1365-2141.2005. 05681.x

47. Riviere E, Saint-Léger M, James C, et al. Platelet transfusion and catheter insertion for plasma exchange in patients with thrombotic thrombocytopenic purpura and a low platelet count. Transfusion. 2015;55(7):1798-1802. doi:10.1111/trf.13041

48. Alias H, Yong WL, Muttlib FAA, et al. Acquired thrombotic thrombocytopenia purpura associated with severe ADAMTS13 deficiency in a 3-year-old boy: a case report and review of the literature. $J$ Med Case Rep. 2018;12(1):276. doi:10.1186/s13256-018-1806-9
49. Roose E, Joly BS. Current and future perspectives on ADAMTS13 and Thrombotic thrombocytopenic purpura. Hamostaseologie. 2020;40(3):322-336. doi:10.1055/a-1171-0473

50. Page EE, Kremer Hovinga JA, Terrell DR, Vesely SK, George JN. Clinical importance of ADAMTS13 activity during remission in patients with acquired thrombotic thrombocytopenic purpura. Blood. 2016;128(17):2175-2178. doi:10.1182/blood-2016-06-724161

51. Jin M, Casper TC, Cataland SR, et al. Relationship between ADAMTS13 activity in clinical remission and the risk of TTP relapse. $\mathrm{Br} J$ Haematol. 2008;141(5):651-658. doi:10.1111/j.13652141.2008.07107.x

52. Peyvandi F, Lavoretano S, Palla R, et al. ADAMTS13 and anti-ADAMTS13 antibodies as markers for recurrence of acquired thrombotic thrombocytopenic purpura during remission. Haematologica. 2008;93(2):232-239. doi:10.3324/haematol.11739

53. Ferrari S, Scheiflinger F, Rieger M, et al. Prognostic value of antiADAMTS13 antibody features (Ig isotype, titer, and inhibitory effect) in a cohort of 35 adult French patients undergoing a first episode of thrombotic microangiopathy with undetectable ADAMTS13 activity. Blood. 2007;109(7):2815-2822. doi:10.1182/blood-2006-02-006064

54. Schieppati F, Russo L, Marchetti M, et al. Low levels of ADAMTS-13 with high anti-ADAMTS-13 antibodies during remission of immune-mediated thrombotic thrombocytopenic purpura highly predict for disease relapse: a multi-institutional study. $\mathrm{Am}$ J Hematol. 2020;1-56.

55. Sui J, Cao W, Halkidis K, et al. Longitudinal assessments of plasma ADAMTS13 biomarkers predict recurrence of immune thrombotic thrombocytopenic purpura. Blood Adv. 2019;3(24):4177-4186. doi:10.1182/bloodadvances.2019000939

56. Pillai VG, Bao J, Zander CB, et al. Human neutrophil peptides inhibit cleavage of von Willebrand factor by ADAMTS13: a potential link of inflammation to TTP. Blood. 2016;128(01):110-119. doi:10.1182/ blood-2015-12-688747

57. Westwood JP, Thomas M, Alwan F, et al. Rituximab prophylaxis to prevent thrombotic thrombocytopenic purpura relapse: outcome and evaluation of dosing regimens. Blood Adv. 2017;1(15):1159-1166. doi:10.1182/bloodadvances. 2017008268

58. George JN. TTP: long-term outcomes following recovery. Hematology Am Soc Hematol Educ Program. 2018;2018(1):548-552. doi:10.1182/ asheducation-2018.1.548

59. Vesely SK. Life after acquired thrombotic thrombocytopenic purpura: morbidity, mortality, and risks during pregnancy. $J$ Thromb Haemost. 2015;13(Suppl 1):S216-S222. doi:10.1111/jth.12953

60. Prevel R, Roubaud-Baudron C, Gourlain S, et al. For the French reference center for thrombotic microangiopathies, immune thrombotic thrombocytopenic purpura in older patients: prognosis and long-term survival. Blood. 2019;134(24):2209-2217. doi:10.1182/ blood.2019000748
Journal of Blood Medicine

\section{Publish your work in this journal}

The Journal of Blood Medicine is an international, peer-reviewed, open access, online journal publishing laboratory, experimental and clinical aspects of all aspect pertaining to blood based medicine including but not limited to: Transfusion Medicine; Blood collection, Donor issues, Transmittable diseases, and Blood banking logistics; Immunohematology; Artificial and alternative blood based therapeutics; Hematology; Biotechnology/nanotechnology of blood related medicine; Legal aspects of blood medicine; Historical perspectives. The manuscript management system is completely online and includes a very quick and fair peer-review system. Visit http://www.dovepress.com/testimonials.php to read real quotes from published authors. 